\title{
Gastrocnemius Kinesiotaping to Improve Dynamic Balance Performance in Middle-Age Healthy Men: Protocol of Randomized Controlled Trial
}

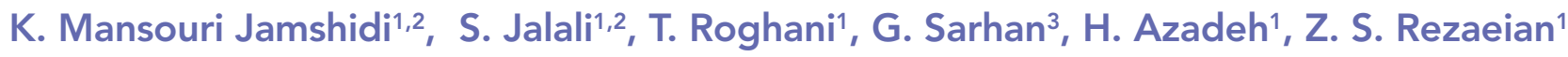 \\ 1 Musculoskeletal Research Center and Department of Physical Therapy, Faculty of Rehabilitation Sciences, \\ Isfahan University of Medical Sciences, Isfahan, Iran \\ 2 Student Research Committee of Rehabilitation Students (Treata), Faculty of Rehabilitation Sciences, Isfahan \\ University of Medical Sciences, Isfahan, Iran \\ 3 Bahrain Physio WWL, Sanad, Bahrain
}

\section{CORRESPONDING AUTHOR:}

\section{Hamid Azadeh}

Musculoskeletal Research Center

Rehabilitation Sciences Research Institute

Department of Physical Therapy

Faculty of Rehabilitation Sciences

Isfahan University of Medical Sciences

Hezar Jarib Avenue

Isfahan, Iran

DOI:

10.32098/mltj.03.2020.11

LEVEL OF EVIDENCE:

Treatment

Kinesio Taping ${ }^{\circledR}$

Comparators

Sham Taping

Patient population

Healthy middle-aged men

\begin{abstract}
SUMMARY
Background. The adhesive taping is supposed to improve joint stability and proprioception and enhance the physical protection in unpredictable situations.

Hypothesis/purpose. The aim of present study was to compare the effect of facilitatory gastrocnemius taping on dynamic balance and muscle activity during Y-balance test in healthy middle-age men.

Study design. Double-blinded (participant, assessor) randomized control trial.

Methods. 30 healthy middle-age men who were not routinely involved in exercise program were randomly assigned in Kinesio Taping® and sham group. They took part in Y-balance test before, immediately, 20 minutes and 24 hours after taping. Y-balance records and medial and lateral gastrocnemius activity were reported for each test. Between group and within group changes were analyzed using independent $\mathrm{T}$ test and repeated measures. Correlation between Y-balance record and gastrocnemius activity were be analyzed using person correlation test.
\end{abstract}

\section{KEY WORDS}

Dynamic balance; Gastrocnemius; kinesiotaping; middle-age.

\section{BACKGROUND}

The adhesive taping is supposed to improve joint stability and proprioception and enhance the physical protection in unpredictable situations (1). Elastic adhesive tape, known as Kinesio Tape (KT), was first introduced by Kenzo Kase (2) and got popular fast (3). KT needs approximately 20 minutes to gain best adhesive strength (2). Several studies have investigated the effects of KT on function and muscle strength and activity using functional tests $(3,4,5,6,7,8,9)$ and surface electromyography (SEMG). Practically, KT-induced changes in muscle activity and the functional measures are time related $(3,5,8,10)$ i.e. in healthy subjects positive effects of KT in muscle activity patterns reached highest level within one hour and then reduced within 24 hours and did not changed up to 5 days (4). Still, the net results of KT on SEMG and functional performance are conflicting: some studies reported that SEMG did not significantly change following inhibitory (1) or facilitatory taping $(11,12)$ in young people. Some authors showed short-term increase in muscle activity following inhibitory gastrocnemius taping (5), while others reported reduced muscle activity of gastrocnemius with inhibitory taping (8). Balance is a critical feature of human posture and movements in daily living. Aging may decrease the dynamic balance capabilities $(13,14)$ and increase falling risk (15); therefore, it may be associated with various musculoskeletal injuries. Since musculoskeletal injuries frequently happen in dynamic activities $(16,17)$ researcher and clinicians are more interested in measuring and screening dynamic balance. The effects of KT on electrical muscle activity and balance have not been well defined in middle-aged subject. Previous studies mostly recruited young adults and athletes under 40 
years old $(3,4,5,6,7,8)$; these people did not suffer from age related balance disorders and fear of falling. Middle-aged people constitute the main society economic power (18). In middle-age (40-65 years) the normal regeneration processes starts to deteriorate as a result of aging process (19). These people are at risk of physical injuries while they are physically and economically active. Beside direct costs, these injuries expose the individual, family and the society to huge indirect costs of unhealthy aging including early retirements and repeated sick leaves. Thus, studying middle-aged subjects are valuable in societies like Iran where the population in gradually moving toward elderly (20).

Gastrocnemius is frequently involved in injuries $(21,22)$; because of its rich proprioception supply $(23,24,25,26)$ gastrocnemius is highly regarded in balance training programs. To improve balance in healthy and injured subjects, physical therapists employ various approaches including adhesive taping. Our aim was to compare the immediate and short-term effects of two types of gastrocnemius kinesiotaping (true facilitatory, sham) on SEMG record of the medial and lateral gastrocnemius and dynamic balance in healthy non-athletic middle-aged men. Our hypothesis was that true KT improves SEMG amplitude (in both gastrocnemius heads) and dynamic balance and these effects become more significant over time.

\section{STUDY AIM}

The aim of this student noncommercial trial was comparison of the immediate and short-term effect of gastrocnemius facilitatory kinesiotaping on electrical activity of the muscle and dynamic balance in healthy non-athletic middle-aged men with control kinesiotaping

\section{METHODS}

\section{Study design}

This study is the protocol of a prospective, double blinded (participant, assessor) randomized, controlled clinical trial of parallel design with two groups. The primary outcome was surface electromyographic record of medial and lateral head of gastrocnemius during Y-balance test. The secondary outcome measure was Y-balance test records in anterior, postero-lateral and postero-medial directions.

\section{Approval and registration}

Institutional ethics committee approved the whole procedure (Ethics Code: IR.MUI.REC.1395.3.814). The study is funded by Isfahan University of Medical Sciences, Vice
Chancellery of Research and Technology (Budget Code: 395814). The protocol has been registered in Iranian Registry of Clinical Trials (IRCT20150131020888N8).

\section{Participants}

The study was conducted at Musculoskeletal Research Center. Participants were healthy, community-based middle-aged men $(\mathrm{N}=30)$, that were recruited through advertisements at urban entertainment districts, March-July 2017. Using non- probability convenient sampling, the volunteers who met inclusion/exclusion criteria of the study were identified according to the medical history and the results of a comprehensive interview and clinical and physical examination by the physical therapist. 40 to 65 years old men were included in the study. The exclusion criteria were any musculoskeletal, cardiovascular and chronic problems that required medication, neurological, vestibular, visual and auditory problems (because of problems in communication with the patient), arthritis or other joint-related problems such as osteoarthritis during the past 12 months, previous allergies to adhesive tapes, previous use of Kinesio Tape or knowledge about its effects (to eliminate psychological effects of taping), regular exercise activities within 6 months prior to and/or during the study, pain in back and lower extremities during the selection process and inability to perform the test.

\section{DATA COLLECTION}

All the subjects who were qualified to participate in the study were informed about study design in detail. They had 48 hours to decide about participating in the study. They were assured that their credentials will going to be saved private. The subjects who agreed to take part in the study were a requested to sign a formal consent.

\section{Pre-randomization evaluation}

Demographic and anthropometric data were collected to make sure that the groups are similar in physical characteristics.

\section{Randomization and allocation concealment}

After signing the inform consent and filling personal data sheet, the participants were randomly assigned into either Kinesio Taping ${ }^{\circledR}$ group $(\mathrm{N}=15)$ or sham group $(\mathrm{N}=15)$ using a coin (simple randomization). A faculty staff that was blind to the study were requested to throw the coin for each subject. The trial was of parallel design (allocation 
ration=1). The subjects were told that they are going to wear tapes for 24 hours but they did not have any clue that what method of taping they are going to experience.

\section{Post-randomization evaluations}

Lower quarter Y balance test records and SEMG data were measured before taping, immediately and 20 minutes after taping (for determining the immediate effect of the tape) and 24 hours after taping (to determine the short-term effect of the tape). In both Kinesio Taping ${ }^{\circledR}$ and sham group.

In healthy subjects, positive effects of KT in muscle activity patterns reached highest level within one hour and then reduced within 24 hours and did not changed up to 5 days (4). Therefore, in present study Lower Quarter Y balance test records and SEMG data will be measured before, immediately, 20 minutes and 24 hours after applying either Kinesio Tape ${ }^{\circledR}$ or sham tape.

\section{TRIAL INTERVENTIONS}

\section{Kinesio Taping ${ }^{\circledR}$ group}

Trained physical therapist (SJ) that was blind to the study method, applied facilitatory Kinesio Tape ${ }^{\circledR}$ on gastrocnemius of dominant leg. The dominant leg was the leg by which the subject hit a soccer ball when the ball was thrown toward him unexpectedly. For facilitatory Kinesio Tape ${ }^{\circledR}$, $15-35 \%$ stretch was applied to therapeutic zone of an "I" strip as proposed by Kase et al. (2). The participant lay in prone; proximal head of KT was anchored under the knee with no tension (tape application in popliteal fossa is not recommended because of the risk of skin irritation (2)). The ankle was fully dorsiflexed by the therapist passively so that gastrocnemius be completely stretched. The Kinesio Tape ${ }^{\circledR}$ was then continued along muscle length and Achilles tendon with $15-35 \%$ tension. The distal one third of the Kinesio Tape ${ }^{\circledR}$ was attached without tension until it ended over the lower surface of heel.

\section{Sham tape group}

According to Gómez-Soriano et al. (5), only the ineffective KT heads (anchor and end) were attached to the back of the calf without continuity. This way, the participants were blind to the intervention they received. They were informed that there is a $50 \%$ chance to receive sham tape but they had no clue that how sham tape looks like. In addition, to make the assessor blind to the study groups, the physical therapist who applied the tapes for either groups covered participant's leg in a disposable cover.
Neither Kinesio Taping ${ }^{\circledR}$ nor sham tape have any side effect. The risk of skin irritation was mentioned and the participants were requested to remove the tape as soon as they feel itching, burning or they find skin rashes around the tape. The subjects did not receive any other intervention during the study in either groups.

In order to make the participants blind to their group, their calf was covered by a disposable sleeve following taping. The same physical therapist (SJ) applied the tapes in both groups. The physical therapist had no role in baseline assessment of inclusion/exclusion criteria, collecting data or analyzing the outcomes. Inclusion/exclusion criteria were assessed before assigning the subjects into study groups (i.e. inevitably the assessor (KMJ) was blinded to study groups). Covered in disposable sleeve, the assessor (KMJ) might not differentiate the study groups. When transferring data into statistic software, the subjects will be coded and the analyzer (KMJ) has no clue to the groups and code.

\section{Blinding strategy}

- The physical therapist who were responsible for primary assessment of the volunteers was informed about the inclusion/exclusion criteria while had no information about the study protocol.

- The selected individuals knew that they will be randomly assigned into one of the study groups and there is 50\% chance of receiving sham tape. They had no clue that how sham tape is different from Kinesio Tape ${ }^{\circledR}$.

- A faculty staff assigned the participant into groups using a coin.

- Employed physical therapist, who had no role in subjects' evaluation, taped subjects in both groups.

- The same researcher who was blinded to study groups, evaluated all the subjects at all assessment sessions.

\section{DATA}

Lower quarter $Y$ balance test records and SEMG data were collected before taping, immediately and 20 minutes after taping (for determining the immediate effect of the tape) and 24 hours after taping (to determine the short-term effect of the tape) (table I).

\section{STATISTICAL ANALYSIS}

The distribution of the data will be determined using Shapiro-Wilk test. In accordance to the distribution of the variables, data will be analyzed using repeated measures/ Wilcoxon signed rank tests in each group. The groups will 
Table I. Data to be collected.

\begin{tabular}{llllll}
\hline Variables & Variable Type & Qualitative & Quantitative & Scale & Measurement \\
\hline Measurement Time & Independent & & Nominal & $\begin{array}{l}\text { Before, immediately, 20 minutes } \\
\text { and 24 hours after taping }\end{array}$ & - \\
\hline Taping Method & Independent & & Nominal & Sham/Kinesio Taping & - \\
\hline Age & Control & Continuous & & Year & Questionnaire \\
\hline Height & Background & Continuous & Meter & Strip Meter \\
\hline Weight & Background & Continuous & Kilogram & Scale \\
\hline Body Mass Index & Background & Continuous & Kilogramxm ${ }^{2}$ & Calculation \\
\hline Lower Extremity Length & Background & Continuous & Meter & Strip Meter \\
\hline $\begin{array}{l}\text { Dynamic Balance } \\
\text { Amplitude of Muscle }\end{array}$ & Dependent & Continuous & & Meter & Lower Quarter \\
Electrical Activity & Dependent & Continuous & & Millivolt & SEMG \\
\hline
\end{tabular}

be compared using Independent $\mathrm{T}$ test/Mann-Whitney U test in SPSS software (IBM Corp. Released 2013. IBM SPSS Statistics for Windows, Version 22.0. Armonk, NY, USA). The $\chi^{2}$-test and Fisher's exact test will be used for categorical variables. Correlation between SEMG records and $\mathrm{Y}$ test results will be determined using Pearson/Spearman correlation test. Mean difference, partial Etta square, effect size and Cohen's d and their respective 95\% CI will be calculated to determine the clinical relevance of the finding. Statistical significance will be considered at $\mathrm{p}<0.05$. The study power will be calculated using $G^{*}$ Power 3.1.5 freeware (University of Düsseldorf, Düsseldorf, Germany) $(27,28)$. The subjects' adherence and the cause for which people left the study or rejected joining the program will be mentioned in consort flowchart (figure 1). Intention to treat analysis will be performed.

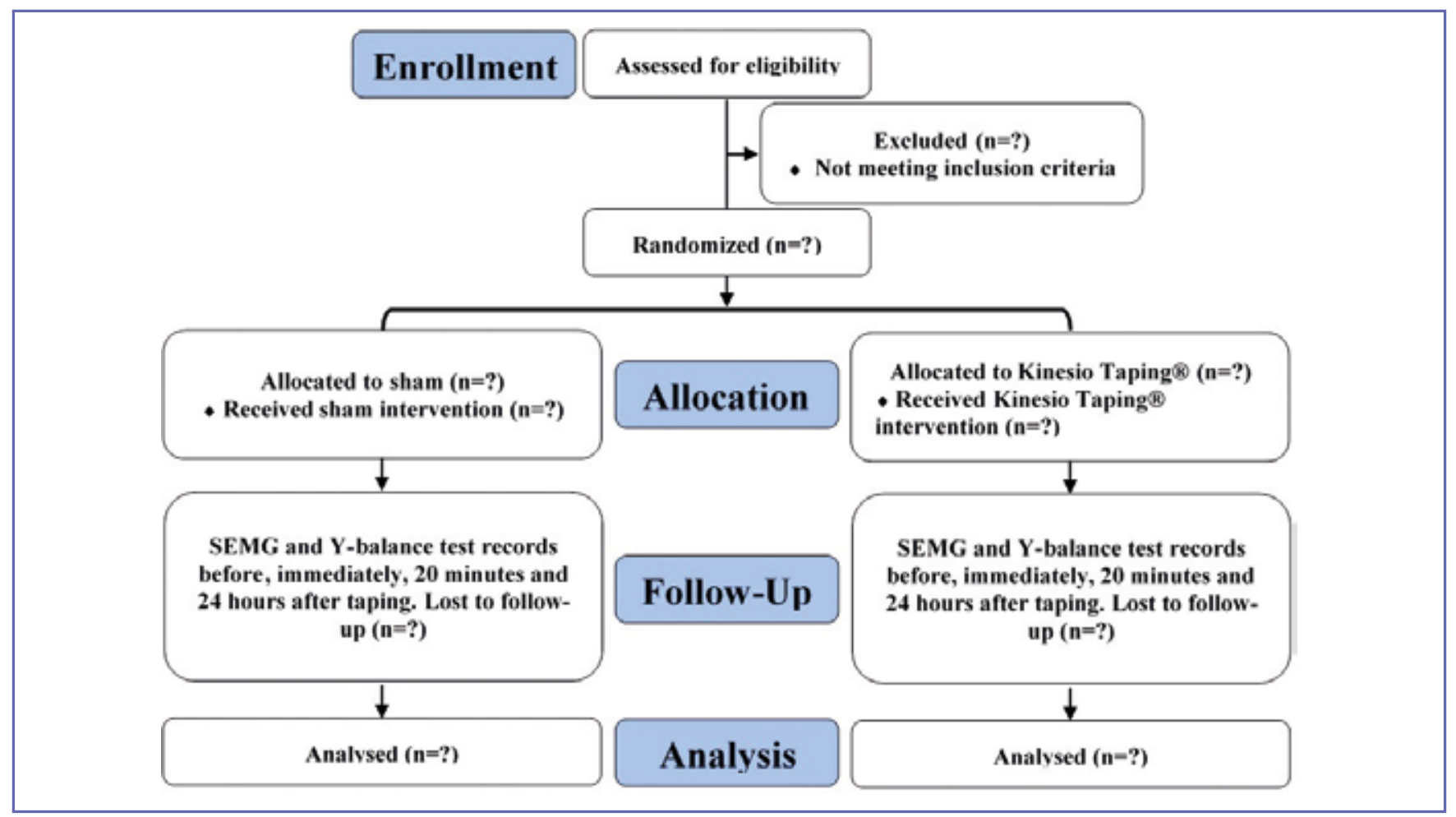

Figure I. Schematic diagram(s) of the trial design, procedures, stages and data collection according to CONSORT. 


\section{Sample size calculation}

There was no similar study to determine the sample size. For pilot studies, 10 subjects in each group is recommended. We considered 15 subjects in either group in present study. Considering $10 \%$ attrition rate, 33 subjects were included in the study.

\section{ETHICAL CONSIDERATIONS}

Institutional ethics committee approved the whole procedure (Ethics Code: IR.MUI.REC.1395.3.814). The protocol has been registered in Iranian Registry of Clinical Trials (IRCT20150131020888N8). KMJ provided detailed information for the participants about the aim and objectives of the study, the possible complications of taping, measurement times and the data confidentiality. Subjects might stop participating in the study any time they want without penalty. Reporting of this trial is done according to the recommendations of the CONSORT statement (29). the study meets the ethical standards of the journal of Muscle, Ligament, and Tendon Journal (30).

\section{TIMELINE}

Ethical approval was obtained in August 2017 from the local medical ethics committee. Subjects were recruited between February-July 2017. All participants completed the study in February 2018. We will finish the statistical analysis by end

\section{REFERENCES}

1. McCarthy Persson U, Fleming H.F, Caulfield B. The effect of a vastus lateralis tape on muscle activity during stair climbing. Man Ther 2009;14(3);330-337.

2. Kase K A, Wallis J, Kase T, et al. Clinical Therapeutic Applications of the Kinesio Taping Method - 3rd Edition. Tokyo, Japan: Ken I kai Co Ltd 2013;12,32.

3. Huang C, Hsieh T, Lu S, et al. Effect of the Kinesio tape to muscle activity and vertical jump performance in healthy inactive people. Biomed Eng Online 2011;10(1):70-81.

4. Wilson V, Douris P, Fukuroku T, et al. The immediate and long-term effects of Kinesiotape ${ }^{\circledR}$ on balance and functional performance. Int J Sports Phys Ther 2016;11(2):247-53.

5. Gómez-Soriano J, Abián-Vicén J, Aparicio-García C, et al. The effects of Kinesio taping on muscle tone in healthy subjects: A double-blind, placebo-controlled crossover trial. Man Ther 2014;19(2):131-136.

6. Nunes G, de Noronha M, Cunha H, et al. Effect of Kinesio Taping on Jumping and Balance in Athletes. J. Strength Cond Res 2013;27(11):3183-3189.

7. Lumbroso D, Ziv E, Vered E, et al. The effect of kinesio tape application on hamstring and gastrocnemius muscles in healthy young adults. J Bodyw Mov Ther 2014;18(1):130-138. of July 2019 and after that we will start the elaboration of scientific paper.

\section{DISCUSSION}

Middle age men are the main human source in economy. ${ }^{18}$ At the same time, gradual progression toward elderly makes them vulnerable to various musculoskeletal injuries. ${ }^{19}$ Balance is an essential part of human function (31). On aspect of rehabilitation is planning for interactive aging (31) i.e. helping individuals to step in seniority in more healthier state. KT is one of the non-invasive, low cost, simply applicable interventions. It may be self-administered by a short training and may be used in conjunction with other approaches to improve their efficacy and lasting effect.

Several studies have investigated the effect of KT on muscle performance and balance in healthy young people and athletes $(3,4,5,6,7,8,9)$ However, its effects in adults and non-athletes are still questionable. Present study seems to be the first study on the effect of KT on balance and muscle activity in healthy middle-aged men. The design of this protocol is such as to minimize bias and bring the results closer to reality. It can also be the starting point for other studies in this age range and in situations where the balance is compromised.

\section{CONFLICT OF INTERESTS}

The authors declare no conflict of interests.

8. Davison E, Andersson C, Ponist B, et al. Inhibitory Effect of the Kinesio Taping ${ }^{\circledR}$ Method on the Gastrocnemius Muscle. Am J sports Sci Med 2016;4(2):33-38.

9. Trecroci A, Formenti D, Rossi A, Esposito F, Alberti G. ShortTerm Delayed Effects of Kinesio Taping on Sprint Cycling Performance. J Strength Cond Res 2019;33(5):1232-1236.

10. Kirmizigil B, Chauchat JR, Yalciner O, Iyigun G, Angin E, Baltaci G. The Effectiveness of Kinesio Taping in Recovering from Delayed Onset Muscle Soreness: A Cross-Over Study. J Sport Rehabil 2019;12:1-28.

11. Lins C, Neto F, Amorim A, et al. Kinesio Taping® does not alter neuromuscular performance of femoral quadriceps or lower limb function in healthy subjects: Randomized, blind, controlled, clinical trial. Man Ther 2013;18(1):41-45.

12. Mak DN, Au IP, Chan M, Chan ZY, An WW, Zhang JH, Draper D, Cheung RT. Placebo effect of facilitatory Kinesio tape on muscle activity and muscle strength. Physiother Theory Pract 2019;35(2):157-162.

13. Lee D, Kang M, Lee T, et al. Relationships among the $Y$ balance test, Berg Balance Scale, and lower limb strength in middle-aged and older females. Brazilian J Phys Ther 2015;19(3):227-234. 
14. Bouillon LE, Baker JL. Dynamic Balance Differences as Measured by the Star Excursion Balance Test Between Adult-aged and Middle-aged Women. Sports Health 2011;3(5):466-469.

15. Talbot L, Musiol R, Witham E, et al. Falls in young, middleaged and older community dwelling adults: perceived cause, environmental factors and injury. BMC Public Health 2005;5(1):86.

16. Cabreira T, Coelho K, Quemelo P. Kinesio Taping effect on postural balance in the elderly. Fisioter e Pesqui 2014;21(4):333-338.

17. Malliaropoulos N, Kakoura L, Tsitas K, Christodoulou D, Siozos A, Malliaras P, Maffulli N. Active knee range of motion assessment in elite track and field athletes: Normative values. Muscle, Ligaments and Tendons Journal(MLTJ) 2015;5(3), 203-207.

18. Eshagh G. Evaluation of social-economic aspects of elderly in Iran. ilam uni Publ 2009;325-348.

19. Thomas D.R. Age-related changes in wound healing. Drugs Aging 2001;18(8):607-620.

20. Fakhredin S. Changes in population age in Iran and its effect on social security protection coverage ratio. Soc Secur Organ 2014;1-3.

21. Armfield D, Kim J, Towers J, et al. Sports-Related Muscle Injury in the Lower Extremity. Clin Sports Med 2006;25(4):803-842.

22. Oberhofer K, Hosseini Nasab SH, Schütz P, Postolka B, Snedeker J, Taylor W, List R. The influence of muscle-tendon forces on ACL loading during jump landing: a systematic review. Muscles Ligaments Tendons J 2017;7(1): 125-135.
23. Fitzpatrick R, Taylor J, McCloskey D. Ankle stiffness of standing humans in response to imperceptible perturbation: reflex and task-dependent components. J Physiol 1992;454:533-47.

24. Lakie M, Caplan N, Loram I.D. Human balancing of an inverted pendulum with a compliant linkage: neural control by anticipatory intermittent bias. J Physiol 2003;551(1):357-370.

25. Loram I.D, Lakie M. Direct measurement of human ankle stiffness during quiet standing: the intrinsic mechanical stiffness is insufficient for stability. J Physiol 2002;545(3):1041-53.

26. Loram I.D, Maganaris C.N, Lakie M. Active, non-springlike muscle movements in human postural sway: how might paradoxical changes in muscle length be produced? J Physiol 2005;564(1):281-93.

27. Kiel C. G Power 3: A flexible statistical power analysis program for the social, behavioral , and biomedical sciences 2007;39(2):175-191.

28. Erdfelder E. Statistical power analyses using $G *$ Power 3.1: Tests for correlation and regression analyses 2009;41(4):11491160.

29. Schulz K, Altman D, Moher D. Consort 2010 statement: updated guidelines for reporting parallel group randomised trials. BMJ 2010;340:c332.

30. Padulo J, Oliva F, Frizziero A, Maffulli N. Muscles, Ligaments and Tendons Journal. Basic principles and recommendations in clinical and field Science Research: 2018 update. MLTJ 2018; 8(3): 305-7.

31. Ross SE, Guskiewicz KM. Examination of static and dynamic postural stability in individuals with functionally stable and unstable ankles. Clin J Sport Med 11/2004;14(6):332-8. 